\title{
Strain Monitoring and Damage Detection of a Filament Wound Composite Pressure Tank using Embedded Fiber Bragg Grating Sensors
}

\author{
S. W. Park ${ }^{1, a}$, \\ D. H. Kang ${ }^{2, b}$, H. J. Bang ${ }^{1, c}$, S. O. Park ${ }^{1, d}$, \\ J. S. Park ${ }^{3, e}$ and C. G. Kim ${ }^{1, f}$ \\ ${ }^{1}$ Div. of Aerospace Engineering, Korea Advanced Institute of Science and Technology, \\ 373-1 Guseong-dong, Yuseong-gu, Daejeon 305-701, Korea \\ ${ }^{2}$ Railway Interoperability Research Team, Korea Railroad Research Institute, \\ 360-1, Woram-dong, Uiwang-si, Gyeonggi 437-757, Korea \\ ${ }^{3}$ Composite Materials Group, Korea Institute of Machinery \& Materials, \\ 66 Sangnam-Dong, Changwon, Kyungnam 641-010, Korea \\ azztwo@kaist.ac.kr, bdhkang@krri.re.kr, ${ }^{c}$ joony@kaist.ac.kr, ${ }^{\mathrm{d}}$ sangfive@kaist.ac.kr, \\ jspark@kmail.kimm.re.kr, cgkim@kaist.ac.kr
}

Keywords: Smart Structure, Fiber Optic Sensor, Fiber Bragg Grating, Filament Winding, Composite, Structural Health Monitoring, Acoustic Emission, Impact response

\begin{abstract}
Composite pressure tanks are rapidly expanding in their range of use. However, for high pressure flammable or toxic gases, there is less confidence about their reliability. In this study, fiber Bragg grating (FBG) sensors embedded into a filament wound composite pressure tank monitored strain and detected damage. The sensor heads and fiber optic lines were protected with an acrylate recoating, adhesive films, and PVC tubes with Teflon ${ }^{\circledR}$ to survive under the harsh environment of the filament winding process. During the quasi-static loading test, the internal strain was measured with embedded FBG sensor arrays. Simultaneously, acoustic emission (AE) signals from matrix cracks and delaminations were detected with both an embedded FBG sensor and an attached piezoelectric sensor. The signal characteristics from the sensors were then compared. For the feasibility test of active sensing, impacts were applied on 3 different positions in each side by an impact hammer. The difference between damaged and undamaged sides in impact response was analyzed. A FBG interrogation system using a wavelength-swept fiber laser (WSFL) was used for the strain measurement, and an EDFA source with a tunable Fabry-Perot filter was used for the high frequency vibration.
\end{abstract}

\section{Introduction}

Recently, with the great demand for lightweight structures, the use of composite materials is widely expanding. Their high specific stiffness and strength make composite materials preferred over metallic parts in various areas and applications. One application, the composite pressure tank, has lately attracted considerable attention due to its high strength and low weight compared to earlier tanks made of metal. The filament-winding method used in composite pressure tanks was developed from military and aerospace use. It was, for example used in such things as missile castings, rocket motors, and fuel tanks. Nowadays, the composite tanks are all around us in our lives. They are used in cars, fire extinguishers, air guns, and many other things. If hydrogen-powered vehicles replace gasoline-powered ones in the future, the demand for these storage vessels will increase dramatically. Although composite materials have many different advantages, they also have diverse damage mechanisms and failure modes that create some difficulties in use [1]. This probable uncertainty becomes even worse in the filament winding process, which is used for making composite pressure vessels. The filament winding process is not fully automatic but instead needs a skilled operator since the fiber tow should be kept from slipping during the helical winding process. In a wet winding process, resin should be wiped out by hand from the fiber tow drawn from the resin bath. Uneven resin content in the composite structure often leaves a lack of continuity in strength and stiffness qualities. 
To overcome this handicap of composite structures, a novel design concept termed the 'smart structure', has been developed by composite material researchers in aerospace engineering. This type of structure allows for a self-monitoring of condition, and also can react against damage by using sensors and actuators. This smart structure warns the user regarding any danger, prevents damage by self-actuation; or, in case of actual damage, can heal the damage itself. While the technology of smart sensing is now maturing and still being extended to other applications, the smart actuation side of the equation still, however, has a long way to go at this point.

Recently, researchers in the smart structure area have been focusing on fiber optic sensors (FOS) for use as sensors in a smart structure. Fiber optic sensors have great advantages, including their small size, high sensitivity and electromagnetic immunity [2]. As one of the fiber optic sensors, a fiber Bragg grating (FBG) sensor utilizes a shift of a Bragg wavelength and has excellent multiplexing capabilities. A good number of past applications highlight its high feasibility for use in composite structures. There have been several attempts to monitor strains with attached or embedded FBG sensors in filament wound structures [1, 3-5].

In this study, in order to apply the smart structure to the composite pressure tanks for better reliability, the feasibility of damage detection with strain monitoring using embedded FBG sensor arrays was tested.

\section{Fabrication of a filament-wound tank}

A STEB was fabricated by filament winding and four FBG sensor arrays were embedded. Each sensor head was recoated with acrylate by the optical fiber recoater (Vytran PTR-100) and wrapped with adhesive films made of epoxy as shown in Fig. 1. This adhesive film reinforced the boundaries of the recoated parts where breakage caused by transverse loading most frequently occurs. In addition, the film increased the visibility of the sensor positions by virtue of its color, while the recoated parts were hardly distinguishable from the other parts of the optical fiber.

Fig. 1 FBG sensor line wrapped with adhesive films.

As shown in Fig. 2, the ingress/egress parts were protected with PVC tubes to prevent them from becoming brittle from the resin flowing out during the curing. To keep the ingress/egress part from breaking during the removal of the moldings, Teflon ${ }^{\circledast}$ film was layered between the fiber lines and the molds. The fiber lines were fixed on the Teflon ${ }^{\circledR}$ film with a thermal-resistant tape. Both ends of each fiber line were reinforced for the connections with an interrogation system as an alternative in case of an internal failure of the optical fiber. Table 1 shows the survivability and characteristics of the four embedded FBG sensor channels. In order to maintain signal stability against transverse loading, FBG sensors with a gauge length of $5 \mathrm{~mm}$ were used in ch. 1 and ch. 2 for helical layers. ch.1 had been broken during the filament winding process by an operator.

Table 1 Survivability and characteristics of the embedded FBG sensors

\begin{tabular}{|c|c|c|c|c|l|}
\hline $\begin{array}{c}\text { Sensor } \\
\text { channel }\end{array}$ & FBG 1 & FBG 2 & FBG 3 & FBG 4 & Embedded Position \\
\hline ch. 1 & $\mathrm{X}$ & $\mathrm{X}$ & $\mathrm{X}$ & $\mathrm{X}$ & Broken during winding \\
\hline ch. 2 & $\mathrm{O}(5,1536)$ & $\mathrm{O}(5,1541)$ & $\mathrm{O}(5,1546)$ & $\mathrm{O}(5,1552)$ & Between 2nd helical layer \& 3rd helical layer \\
\hline ch. 3 & $\mathrm{O}(10,1552)$ & $\mathrm{O}(10,1546)$ & $\mathrm{O}(10,1541)$ & $\mathrm{O}(10,1536)$ & Between 2nd hoop layer \& 3rd hoop layer \\
\hline ch. 4 & $\mathrm{O}(10,1541)$ & $\mathrm{O}(10,1546)$ & & & Between 3rd hoop layer \& 4th hoop layer \\
\hline
\end{tabular}




\section{Experimental setup}

Two types of FBG sensor systems were used to measure both the strain and damage signal simultaneously. A Fiberpro IS7000, a commercial FBG interrogation system using a wavelength-swept fiber laser (WSFL), was used for the strain monitoring of the multiplexed FBG sensor channels. Usually, a WSFL laser has more power than other types of interrogation systems, thus a greater number of multiplexed FBG sensors can be connected and it enables measurement using FBG sensors with reduced reflectivity by transverse loading. Because FBG sensors and optic fiber lines inside a filament-wound tank experience a certain amount of transverse loading which can possibly cause a reduction of reflectivity, an interrogation system with a high power laser source is necessary. The WSFL laser source can be a viable solution for the monitoring of the structural integrity of filament-wound composite tanks.

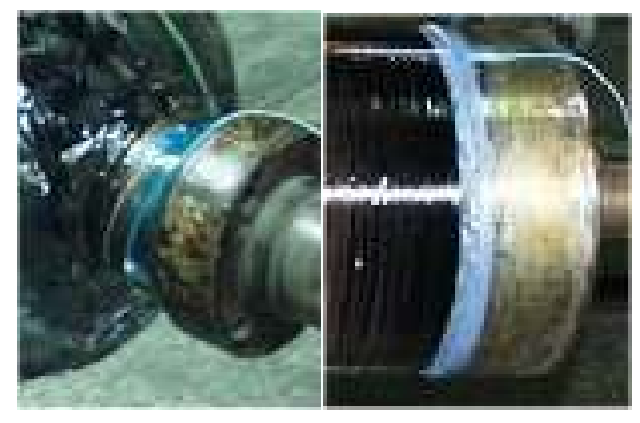

Fig. 2 Ingress/egress parts protected with PVC tubes and Teflon ${ }^{\circledR}$

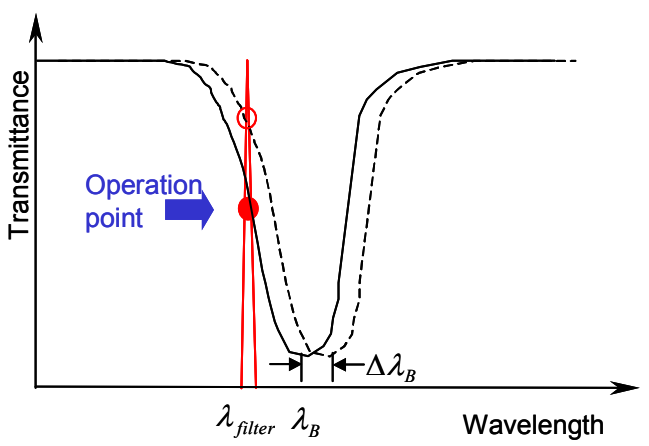

Fig. 3 Intensity-based demodulation of the FBG sensor signal.

The second FBG sensor system measured high frequency vibration with a FBG sensor using a tunable Fabry-Perot filter with a narrow free spectral range (FSR). By controlling voltages on the Fabry-Perot filter, the filtered wavelength was located at the most linear position in the reflection spectrum of the FBG sensor, as shown in Fig. 3. According to the change in the Bragg wavelength $\lambda_{B}$ by incident strain from a high frequency vibration, the intensity of the filtered signal varied linearly with the amount of wavelength shift $\Delta \lambda_{B}$. However, to maintain a uniform sensitivity, the operation point continually followed $\lambda_{B}$ with a relatively slow wavelength shift caused by the applied strain from loads, temperature, and other factors. This intensity-based demodulation system used a photodetector with a $125 \mathrm{MHz}$ bandwidth (Thorlabs PDA-400), and the sensitivity was verified by a forced actuation test using a rectangular piezoelectric actuator (Fuji Ceramics C-82) bonded with a FBG sensor. The sensitivity was measured between 2.49 and $2.82 n \varepsilon_{r m s} / \sqrt{H z}$ at $100 \mathrm{~Hz} \sim 5 \mathrm{kHz}$ vibration [6]. Sensitivity at this level was high enough to detect an AE signal induced by failure in composite beams and plates.

In this research, feasibility of damage detection was examined using embedded FBG sensors in the composite tank. Two possible techniques were adopted from both passive and active sensing methods, quasi-static loading test and impact response test. In both tests, a disk-type piezoelectric sensor (Fuji Ceramics C- $6,2 \times 5 \mathrm{r} \mathrm{mm}$ ) was attached on the surface of the tank for damage detection at the same location as the embedded FBG sensor. Voltage signals from the piezoelectric sensor and the photodetector were acquired by a digital storage oscilloscope (Tektronics TDS-420) and a data acquisition board (National Instruments PCI-6110E) installed on a personal computer system. The Labview software used was programmed to maintain sensitivity at the maximum point by controlling the tuning voltage of the Fabry-Perot filter. The software also recorded the high frequency vibration signals of both the piezoelectric sensor and the FBG sensor, by triggering a piezoelectric sensor signal that was expected to have better sensitivity. 
Fig. 4 shows the filament-wound tank fixed in an $x-y-z$ moving jig for the quasi-static loading test. Load was applied on the surface of the tank where $15 \mathrm{~mm}$ away from the sensors were applied. A ball-shaped indenter with a point contact produced a quasi-static load by rotating a wheel. The load was increased until several damage signals had been detected. Strains of FBG sensor ch. 3 were measured with the load and AE signals from the piezoelectric sensor and the FBG sensor were measured. Then, spectrums of two signals were compared with the fast Fourier transform (FFT) of measured data. Then, as a first step for the active sensing of composite tanks, the difference was examined between impact responses caused by the damage made during the quasi-static loading test. The impacts were applied on six positions at three different distances from the sensors in both sides and repeated five times for each position. Fig. 5 shows the positions of damage, sensors and impact points. The voltage signal from the piezoelectric sensor located on the tip of the impact hammer was also recorded with the sensor signals. The signal was used for triggering and also keeping from the improper impacts of too small, too big or double excitation.

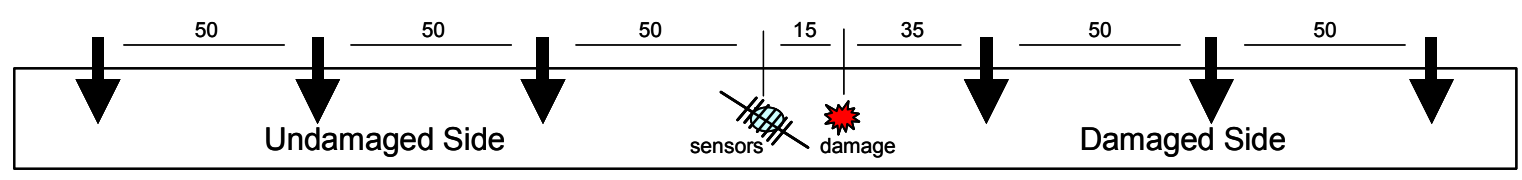

Fig. 5 Positions of damage, sensors and impact points.

\section{Results and discussions}

Quasi-static loading test was carried out until the maximum strain was about $0.3 \%$ strain estimated from the tuning voltage of Fabry-Perot filter. During the test, internal strains of a hoop layer were monitored once a second from the FBG ch. 3 as shown in Fig. 6. Also, the AE signals from damage were detected by both piezoelectric and FBG sensors simultaneously. However, while the piezoelectric sensor caught more than twenty damage signals, FBG sensor could not detect any meaningful signals. Even there were a lot more AE signals skipped for their low amplitude under the threshold level of the piezoelectric sensor. Fig. 7 shows typical looks of FFT results from piezoelectric and FBG sensor signals. The piezoelectric sensor showed enough sensitivity in this composite tank case but FBG result was difficult to be assumed it represented AE signal from damage by its low signal to noise ratio. The reason of such results can be expected in two ways. First one is the relatively low sensitivity of the high frequency FBG sensor system comparing to the piezoelectric sensor. The other assumption is the different attenuation rates between the surface and the medium from difference of the acoustic wave propagation modes. Considering the primal purpose of using embedded FBG sensors in composite tanks, FBG sensor system of higher sensitivity is needed for the detection of damage occurring from matrix cracks delaminations under quasi-static loading.

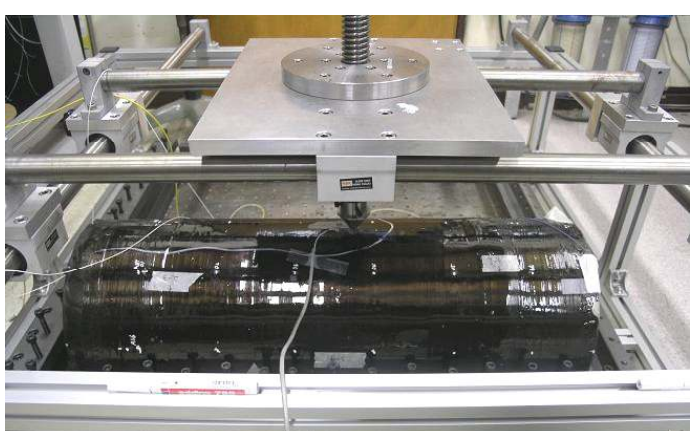

Fig. 4 Experimental setup of the quasi-static loading test.

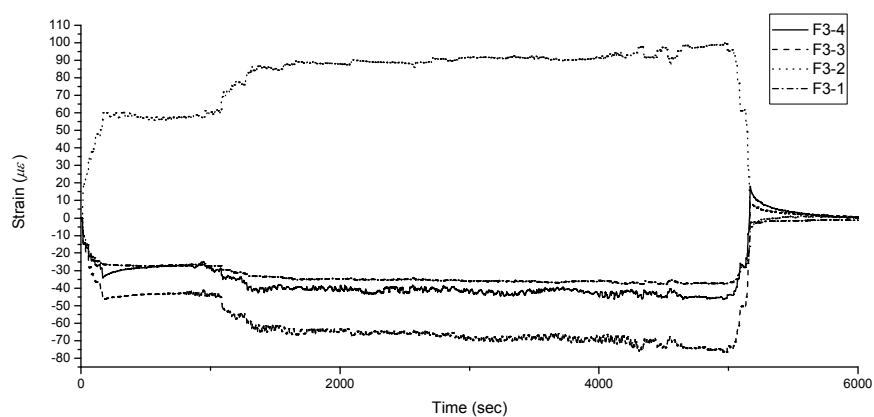

Fig. 6 Strain measured from FBG ch. 3during the quasi-static loading test. 


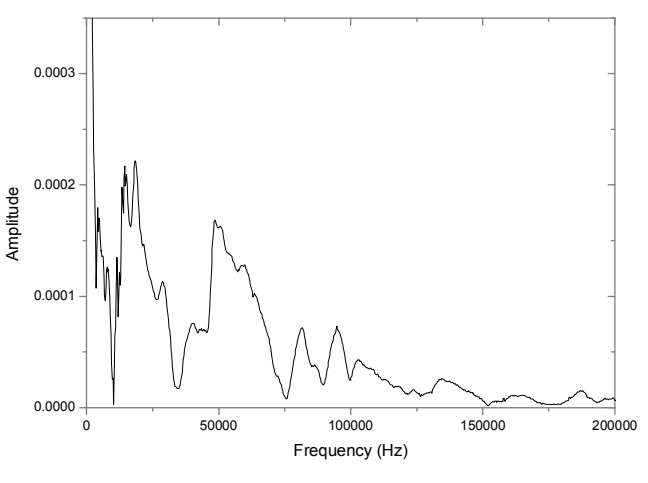

(a) FFT of piezoelectric sensor

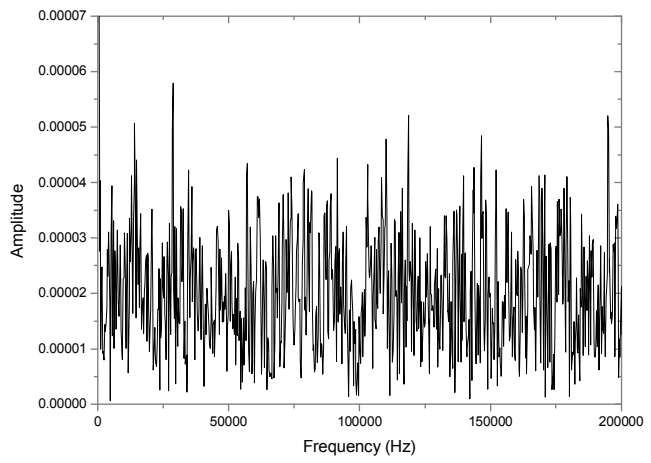

(b) FFT of FBG sensor

Fig. 7 FFT of piezoelectric and FBG sensor during the quasi-static loading test.

On the other hand, the FBG sensor could properly measure the impact responses with the piezoelectric sensor. In addition, this impact test showed the possible solution of damage detection in composite tanks. From the results shown in Fig. 8, the obvious difference was noticeable between the impact response signals of damaged and undamaged sides. Regardless of the type of sensors and the distance, certain ranges of the impact response signal in the frequency domain showed common decreases in the damaged side while the other parts showed exact coincidence. Near $6 \mathrm{kHz}$ (highlighted parts), listed six figures have the same decrease though the levels of amplitude are all different. This difference is quite encouraging in the viewpoint of active sensing for composite tanks. The information from the difference of spectrums by different impact levels or at different impact locations enables the damage assessment using system identification skills.
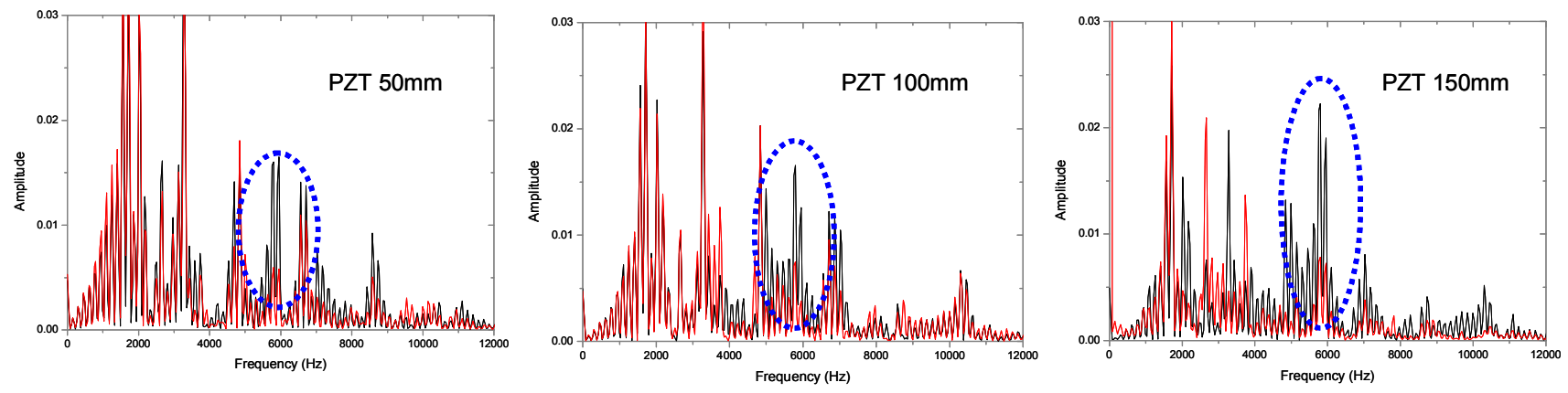

(a) FFT of piezoelectric sensor
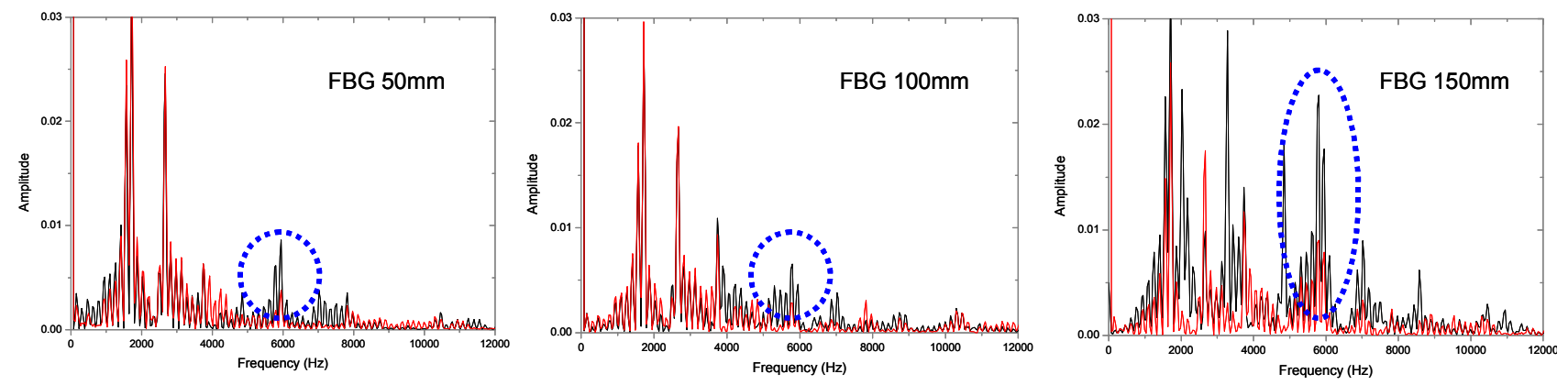

(b) FFT of FBG sensor

Fig. 7 FFT results of damaged (gray) and undamaged (black) sides at each distance.

However, such an active sensing method essentially needs external excitation for the response signal. In the viewpoint of the smart structure, this method can be regarded as incongruent way of damage detection in operation. If we think of this composite tanks can be used for the hydrogen storage of next generation cars, we could get regular inspections whenever we fill the hydrogen or change oils. Also, random vibrations in the moving vehicles can be the excitation sources for the 
continuous damage detection in operation and then embedded FBG sensors can used for damage monitoring as well as their primal purpose, strain monitoring.

\section{Conclusions}

In this study, A standard testing and evaluation bottle (STEB) was fabricated with embedded FBG sensor arrays. The sensor heads and optical fiber lines were protected to survive under the harsh conditions of the filament winding process. To verify the feasibility of damage detection using the sensors, a quasi-static loading test and an impact test were performed using the high frequency FBG sensor system with the controlled tunable Fabry-Perot filter, and the internal strains were measured simultaneously by the FBG interrogation system using WSFL during the loading test. AE signals from damage and impact responses at different distances were measured using both an embedded FBG sensor and an attached piezoelectric sensor, and the signal characteristics were compared by fast Fourier transform. The attached piezoelectric sensor could successfully detected AE signals during the loading test, but the embedded FBG sensor did not have enough sensitivity for damage detection in this case. In the impact test, both sensors were adequate for comparing the impact responses at $50 \sim 150 \mathrm{~mm}$ distances in both damaged and undamaged sides. The damage caused decreases in amplitudes of impact response spectrums at the same ranges regardless of distances or types of sensor. This result showed the damage assessment using embedded FBG sensors was possible from the regular or continuous analysis of impact responses in the filament wound structures.

\section{Acknowledgement}

This paper was performed for the Hydrogen Energy R\&D Center, one of the 21st Century Frontier R\&D Program, funded by the Ministry of Science and Technology of Korea.

\section{References}

[1] R. C. Foedinger, D. L. Rea, et. al.: Proc. SPIE Vol. 3670 (1999), p. 289.

[2] R. M. Measures and K. Liu: IEEE Circuits Devices Mag. Vol. 8(4) (1992), p. 37.

[3] J. Degrieck, W. De Waele, P. Verleysen: NDT\&E Inter. Vol. 34 (2001), p. 289.

[4] H. K. Kang, J. S. Park, D. H. Kang, C. U. Kim, C. S. Hong and C. G. Kim: Smart Mat. and Struc. Vol. 11(6) (2002), p. 848.

[5] D. H. Kang, C. U. Kim, S. W. Park, C. S. Hong and C. G. Kim: Proc. ACCM-4 (2004).

[6] H. J. Bang, S. M. Jun and C. G. Kim: Measurement and Sci. Tech. Vol. 16(3) (2005), p. 813. 